\title{
AUTOMATED FISH FINGERLINGS COUNTER SYSTEM: AN EVALUATION OF IMAGE SEGMENTATION ALGORITHMS IN OVERLAPPING OBJECTS
}

\author{
Nel R. Panaligan \\ Faculty, Computing Department, Davao del Sur State College, Digos City, Philippines
}

\begin{abstract}
The development of automatic fish counters had been driven by the need for accurate, long-term and costeffective counting and recognition for the advancement of aquaculture in the Philippines. Non-invasive methods of fish counting are ultimately limited by the properties of the immerging technologies like when candidates for counting are transparent and or small (Bangus Fry). Image processing is one of the most modern approaches in automating the counting process. The main objective of the study is to evaluate three image segmentation algorithms namely (1) Watershed Algorithm, (2) Hough Transform, (3) Concavity Analysis, in 2D image, whether or not they are capable of segmenting two-weeks old bangus fry's' in an image. The basic steps involved in the conduct of this study are the following; Image acquisition, Image Pre-Processing, Image segmentation, and Object counting. Result shows that the first method of the Concavity Analysis which locates the contours or curve points edges of the objects in an image performs best with the other algorithm with an accuracy rate of $94.36 \%$ with 7 false positive detections, and 154 False Negative, in an experimental data of four sets of 2D image ranging from 100, 200,300, and 400 bangus fry per test image.
\end{abstract}

Keywords: Aquaculture, Image Segmentation Algorithms, Watershed Algorithm, Hough Transform, Concavity Analysis, Evaluation

\section{INTRODUCTION}

Fish product contributes a significant amount of protein demand of human nutrition and its consumption have dramatically increased - about 27 million tons of fish were consume during 1948 and this has increase to about 145 million tons during 2007 (Philippine Export 2015-2017). In a study conducted by Dowlati et al which was mentioned by Gana et al, Fish product is about 16\% of human diet all around the world (Aliyu et al., 2017). With vast growing sector in the economy of the Philippines, fishing plays a major role in Agriculture, Hunting, Forestry and Fisheries (AHFF) are among of the second largest contributors of the GDP at 34 and 9.2\% of the economy. The Philippine economy prides itself in having been resilient during the global financial crisis of 2008, until now, the Bangko Sentral ng Pilipinas (BSP) says that the country has a well-insulated economy, characterized by steady remittances, robust private consumption and capital formation, and lastly, a well-maintained services sector and a booming industrial base.

Considering the contribution of the fishing industry in the country, it remains a question why the fish farmers (i.e. Bangus) in the country remained in indigenous way of handling their farms, specifically in counting the fingerlings, feeding, and classifying (i.e. size and weight). This type of farming is also laborious, especially the counting of fingerlings to sell for interested buyers, where people find it hard to obtain the exact number of fishes because of frequent human error, behavioural aspects of fry also affects the method of counting as workers are easily distracted and tend to forget the total count as it accumulates later which contributes to losses, not only for fish farmers whether small or large scale, but also for the customers.

To address the needs for a highly reliable approach necessary in the field of fisheries and aquaculture, it is vital to develop and utilize a mostly reliable method of counting the fry in a short period of time without compromising reliability. Artificial intelligence

This research paper will assess or evaluate three (3) image segmentation algorithms namely Watershed Algorithm, Concavity Analysis, Morphological Transformation in bangus fry counting, where common scenario of multiple 
overlapping occurred. The evaluation will be based on; Time of completion per test, Accuracy rate based on total number of detected fry

\section{REVIEW OF RELATED LITERATURES}

\section{Automated Fish Fry Counting and Schooling Behaviour Analysis Using Computer Vision}

In this paper the authors developed a system that claims to be able to count a fish fry with accuracy rate of $95 \%$. The study uses Edge Detection Algorithm and Binarization, two common algorithm used in computer vision. In this study, the method of counting is by detecting the pixel area occupied by each fish silhouette, for every fish frame, the total fish count is the sum of the area inside every contour. In an experimental data of 200, 400, 500, and 700 fish fry in image, the accuracy rate was considerably high, but as the number of fry in image increases the accuracy rate decreases. The decreasing of the accuracy rate was due to poor segmentation of the occluded or overlapping of fry's in the image(Labuguen et al., 2012).

\section{Blood Cell Image Segmentation Based on the Hough Transform and Fuzzy Curve Tracing}

Noise and uneven brightness in an image can cause inaccurate result in computer vision. In this paper, researchers proposed a new method of counting blood cells in an image, they used Hough Transform and Fuzzy Curve tracing in an image to identify each blood cell present in image. Their proposed method performs better in comparison to the other two algorithms in this study, their method, the rough cell regions of the cell has been detected as closed circles using the Hough Transform Algorithm. However, their proposed method has a main limitation, particularly on the shapes, the algorithms assumes that the shape of a cell is always in circle, thus, cells that has a heavy curve on each side even when Fuzzy curve tracing is applied detects the cell poorly, or even worst skips them in counting process(Kong \& Engineering, 2011).

\section{A Fish Population Counting Method using Fuzzy Artificial Neural Network}

One challenging area in the field of computer vision particularly in image processing is the overlapping or occlusion of objects that needs to be counted. In this paper, the authors present an efficient methodology that claims to get a fish count in image. Fuzzy Artificial Neural Network was used in this study, this method used fuzzy theory to turn the fish pictures into multiple layers of binary data set and uses neural networks to process the degree of memberships (Zheng \& Zhang, n.d.).

\section{RESEARCH DESIGN AND METHODS}

The image segmentation algorithms that has been selected was evaluated with the use of $2 \mathrm{~d}$ image taken in a specially designed container, the iteration of testing per algorithms will be limited to four times. Python and opencv was used in evaluating the algorithms. Result has been evaluated with the following parameters; time of completion per test, accuracy rate, and total number of detected fry with multiple overlapping fingerlings. Each of the image segmentation algorithms was compared through the mentioned result evaluation criteria where observations have been conducted.

\section{A. CONCEPTUAL FRAMEWORK}

In this framework, Image Pre-processing consists of contrast enhancement of the 2D image to ensure the evaluation of the image quality which will make the fingerlings distinguishable from other objects and the background before having it denoised. The denoising stage ensures that any unnecessary noises in the image has been removed for better image that has been binarized. The second phase of framework is the application of the Image segmentation and Normalization of the image, lastly was the Prediction phase.

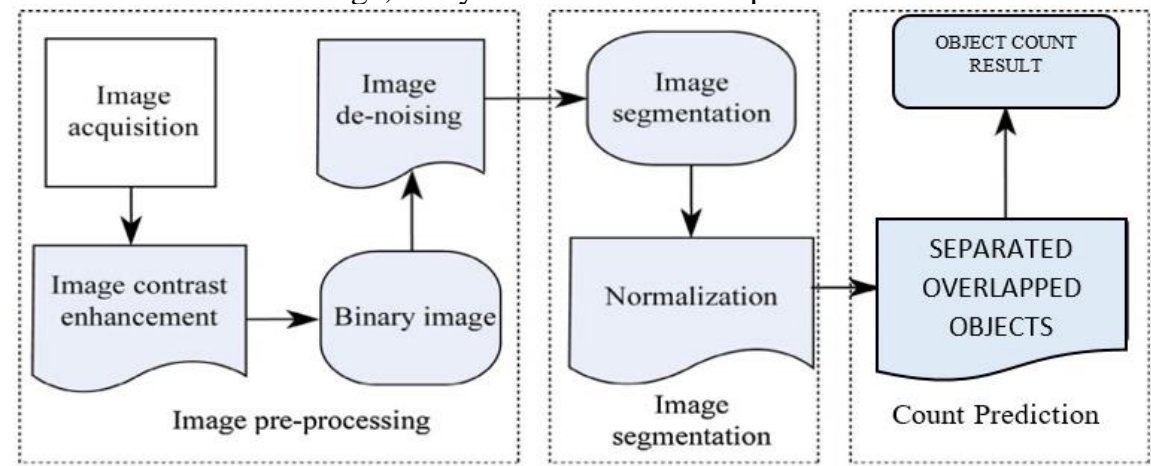

Fig 1. Adopted Framework from (Zhao, Cao, \& Tu, 2014) 


\section{B. IMAGE ACQUISITION}

In taking 2D image, Logitech c270 HD USB-Webcam which is a High Definition camera with 720 pixels mounted $35 \mathrm{~cm}$ above the aquarium was used. The camera was directly connected to Laptop with 8GB RAM, Core i7 Processor with $3.2 \mathrm{GHz}$ for the processing. The aquarium size was $40 \times 30 \mathrm{~cm}$ and $20 \mathrm{~cm}$-depth; it was used as the base where the lighting was placed underneath it in order to have greater contrast of the fry. Above it was a $42 \times 30 \mathrm{~cm}$ in size with $5 \mathrm{~cm}$ depth served as the container where the fry was placed. The water inside the container was $1 \mathrm{~cm}$-deep; this was intended in order to avoid heavy overlapping of the fry.

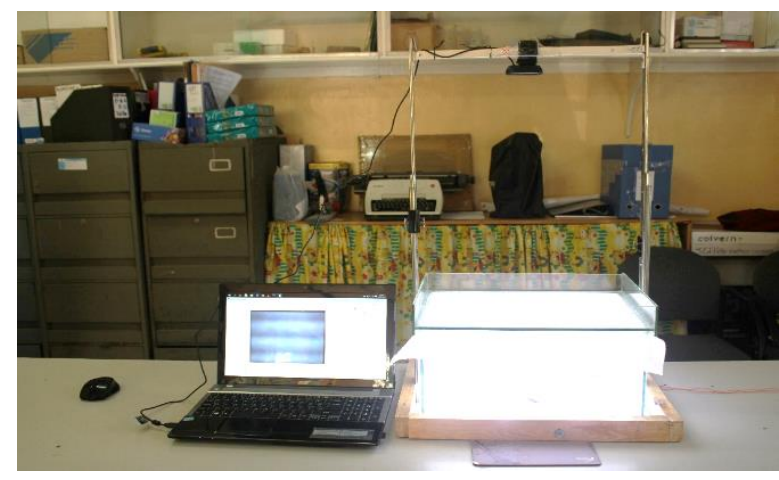

Fig 2. Prototype Set-up

\section{Image Segmentation Algorithms}

Python Image Library (PIL) and OpenSource Computer Vision (OpenCV) among others are the main image manipulation and processing libraries was used in this study, as they carry out most effectively in terms of image processing techniques. Jupyter Notebook was the Integrated Development Environment was used in this study.

\section{C.1. Watershed Algorithm}

In this study, the algorithm was invoked by calling the skyimage.morphology module and importing the watershed algorithm. Otsus Thresholding Method which returns the Otsus edge detection algorithm was used in order to separate the background and foreground, the study applies two methods of segmenting the objects in the image. The first method was Euclidian Distance Transform (EDT): this measures the number of fish directly, and the idea was to idea was to create a border as far as possible from the centre of the overlapping objects. The second method used in this algorithm was done by flooding the local maxima. It has been achieved by computing the distance of the background. The local maxima of this distance (the minima of the opposite of the distance) are chosen as markers, and the flooding of basins from such markers separates the overlapping objects along a watershed line.

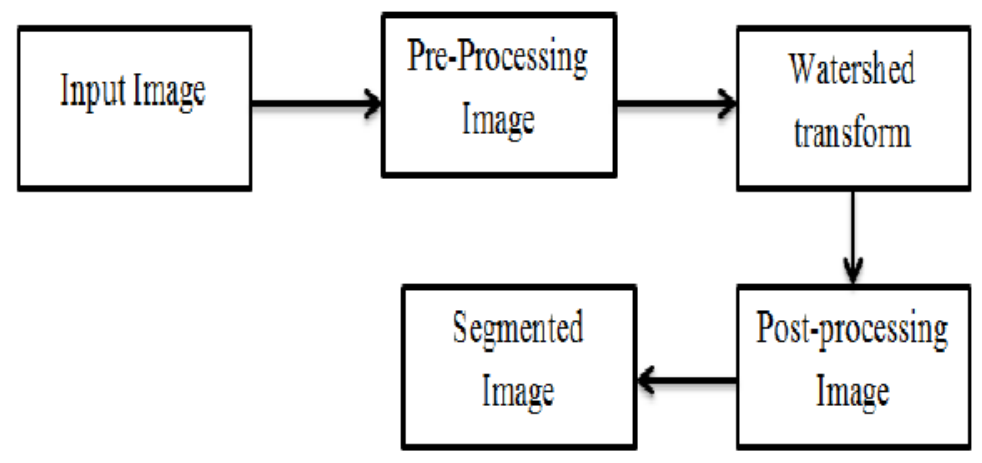

Fig. 3 Block Diagram of Watershed Algorithm 
WATERSHED ALGORITHM IN 2 METHODS

Number of fish (method 1): 113 Number of fish (method 2): 180

Overlapping objects

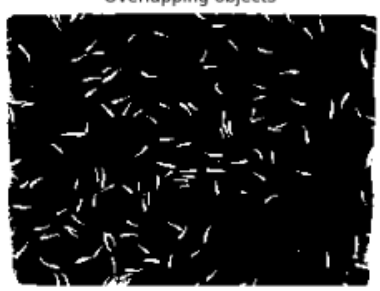

Distances

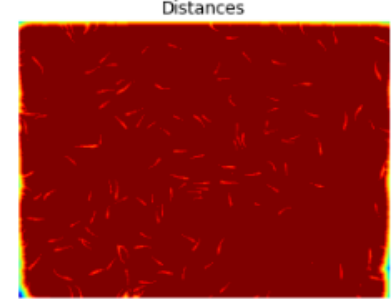

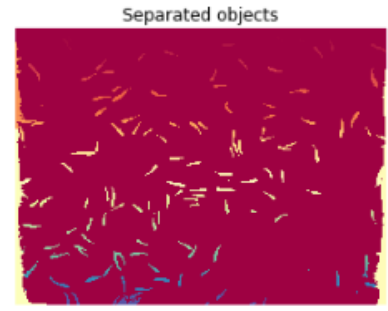

--- 0.38725709915161133 seconds ---

Fig. 4 Sample Result with Count of Fish Detected and Processing Time

Figure 3 shows the Block diagram to easily understand how the algorithm in deteting the objects in the image. Figure 4 on the other hand shows a sample result of the algorithm.

\section{C.2. Concavity Analysis}

This algorithm locates the contours or curve points edges of image in an image. In order to get a better segmentation result, contour parameters has been made. The parameters for contours have been used for locating necessary points of the candidates in the image. Iteration for contours with ellipse fitting has been made in order to fully detect the pixels that represent the point and centroid of the object.

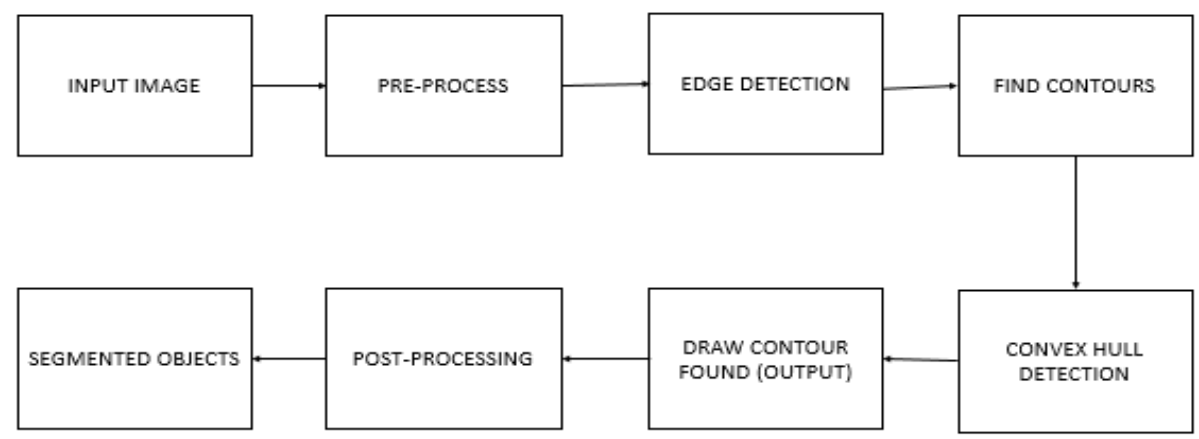

Fig. 5 Block Diagram of Concavity Analysis

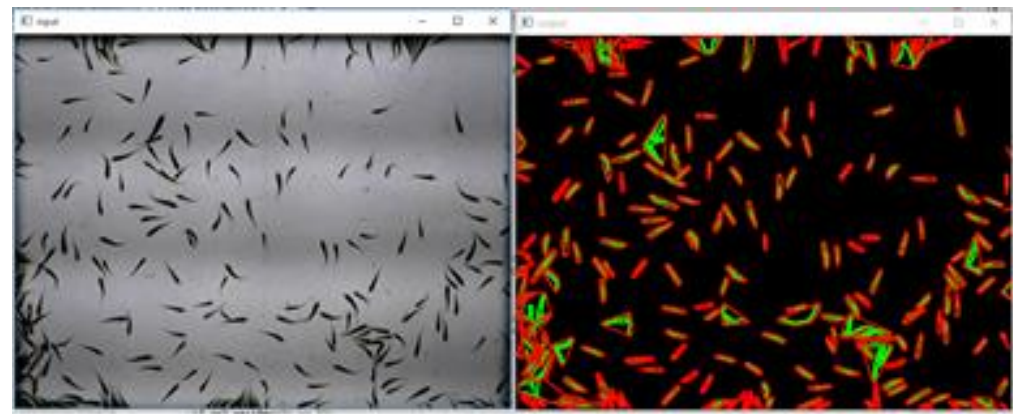

Fig. 6. Sample Result Image

Figure 5 shows how the algorithm works particularly in identifying objects in the image. It starts by pre-processing the image particularly image binarization, an essential step to clean the image. Then it uses edge detection algorithm to further ease the identification of objects in the image before it segments the identified objects for counting. 


\section{Impact Factor 7.39 ㄷ Vol. 11, Issue 1, January 2022 \\ DOI 10.17148/IJARCCE.2022.11103}

\section{C.3. Morphological Transformation using Opening and Closing Mathematical Morphology}

This algorithm is derived from fundamental operation of Erosion and Dilation; however, it is less destructive than erosion in general. In this project the algorithm has been by applied by first applying or looking for the best threshold value for the image to be binarized through trial and error, then create a kernel with square image value for erosion. Then call values for threshold and kernel during erosion for refining all edges in the binary image. In figure 8 , it can be observed that bangus fry which clumps together at the near edge of the container, once binarized tends to be treated as one dark object or blob in the image, which significantly affects the total number of candidates detected by the algorithm since they were counted as one, unlike with the others which slightly touched or overlapped. See Figure 7 for the block diagram of the algorithm which in detailed shows the process how the algorithm works.

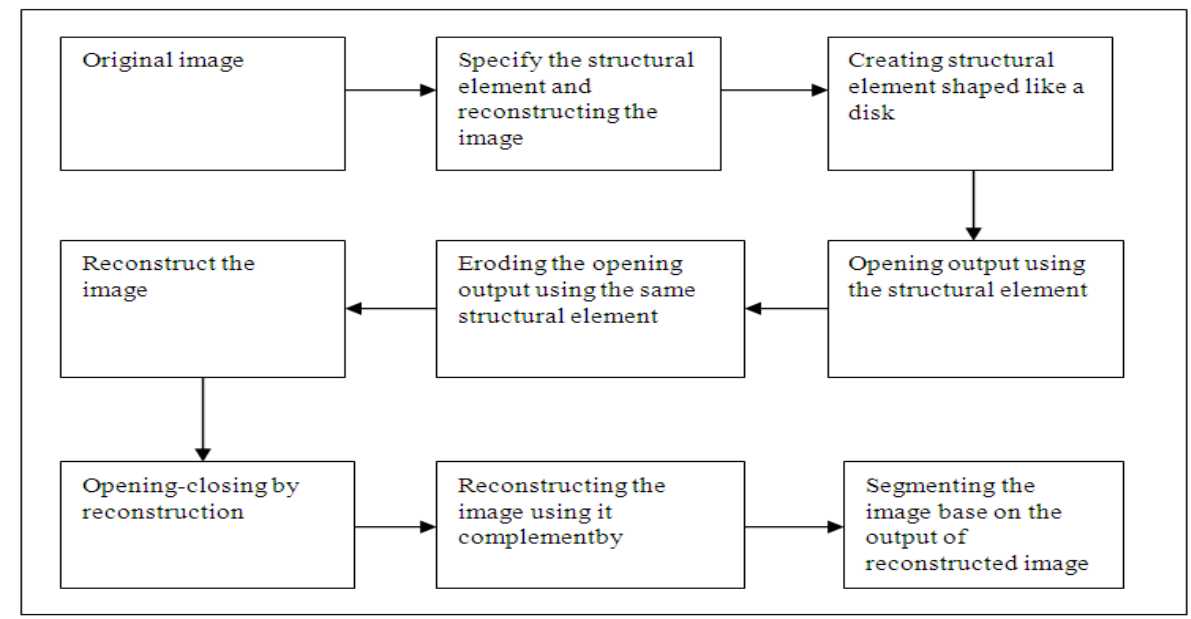

Fig 7. Block Diagram of the Algorithm
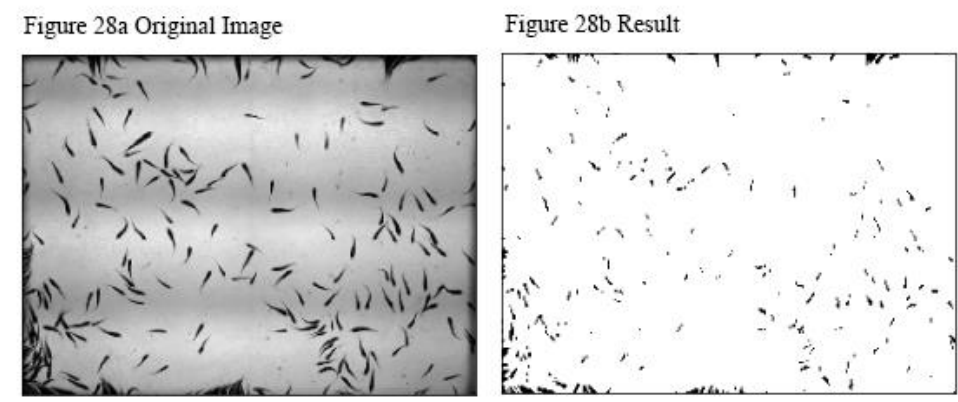

Number of fish detected: 300 --- 0.2021334171295166 seconds ---

Fig 8. Sample Result Image

\section{RESULTS, CONCLUSSIONS, AND RECOMMENDATION}

\section{A. RESULTS}

In this study, the researchers focused on evaluating three image segmentation algorithms namely; Watershed Algorithm, Concavity Analysis, Morphological Transformation. Percentage of Accuracy, and the total count for False Positive and False Negative were used to determine what algorithm performs best. The formula used in this study for getting the accuracy was:

Percentage $(\%)$ of Accuracy $=$ Predicted count $/$ Total Number of Fry $* 100$

While,

False Positive in this study is the total number of incorrectly identified fry.

False Negative in this study is the total number of incorrectly rejected fry.

Table I shows the summarized report of the algorithms in a set of 2D image with bangus fry's ranging from $100-400$. It narrates which particular algorithm based on this study performs well in counting and segmenting occluding objects in the image. 
Impact Factor $7.39 \div$ Vol. 11, Issue 1, January 2022

DOI 10.17148/IJARCCE.2022.11103

TABLE 1. Summary Report of Accuracy Rate per Method and Total False Positive and Total False Negative Per Algorithm.

\begin{tabular}{|l|l|l|l|l|l|l|}
\hline \multirow{2}{*}{ ALGORITHM } & \multicolumn{2}{l}{$\begin{array}{l}\text { \% ACCURACY } \\
\text { METHOD }\end{array}$} & \multicolumn{2}{l}{$\begin{array}{l}\text { TOTAL FALSE } \\
\text { POSITIVE }\end{array}$} & \multicolumn{2}{l|}{ TOTAL FALSE } \\
NEGATIVE
\end{tabular}

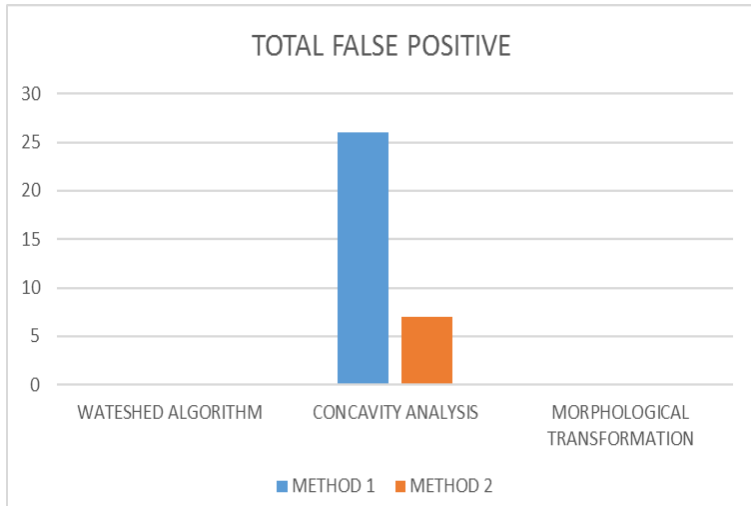

Fig 10. False Positive

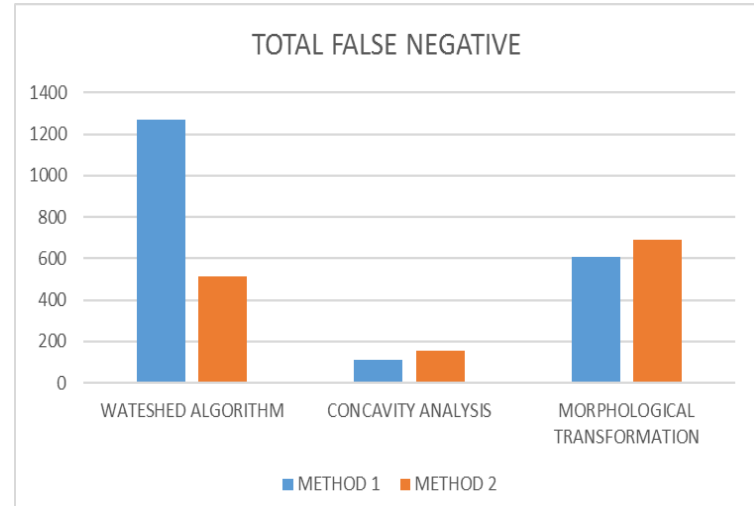

Fig. 11 False Negative

Figures 10 and 11 shows' in detail the levels of False Positive detection and False Negative per algorithm. This further supports the claim that based on the accuracy rate of the second method of the watershed algorithm. The detection rate of each algorithm in this study relied on user defined values for its parameters in automatic detection. The most accurate algorithm based on the performance of its detection is sequenced hierarchically below in terms of accuracy rate of detection with no false positive detection;

1. Second combination values for Concavity Analysis with general average of $94.36 \%$ accuracy with a total of 7 false positive detections, and 154 false negative detections. This algorithm uses threshold values of $(\mathrm{L}=80 \mid \mathrm{U}=180)$.

2. First combination values for Concavity Analysis with general average of $95.96 \%$ accuracy with a total of 27 false positive detections, and 111 false negative, this algorithm uses threshold values of $(\mathrm{L}=80 \mid \mathrm{U}=170)$.

3. First Combination Values for Morphological Transformation with a general average of $83.85 \%$ accuracy with 0 false positive detection. This first combination used the values of $(\mathrm{Th}=(5) \mid \mathrm{K}=(2,2))$. It yields a total of 606 false negative detections.

4. Second Combination Values for Morphological Transformation with a general average of $100.37 \%$ accuracy with 108 false positive detections, and 67 false negative. This first combination used the values of $(\mathrm{Th}=(7) \mid \mathrm{K}=(3$, 1)).

5. Second Method applied in Watershed Algorithm identifying the Local Maxima is the most effective with a general average of $86.47 \%$ accuracy, although this method has a total of 515 false negative detections still it outperforms the rest of the methods in every algorithm since it yields 0 false positive detection. This method has a value of $(6,7)$ for the Threshold used in identifying and cleaning the tiny intersections between the fishes.

6. First Method applied in Watershed Algorithm which uses the EDT with a general average of $70.81 \%$ accuracy with 0 false positive detection. This method, directly measures the number of fish by directly using the distance map. This method is one of the classic way of separating touching objects in binary images, the idea is to create a border as far as possible from the center of the overlapping objects, in this case the fingerlings. This method uses the $(0.1)$ value for its distance. This method yields a total of 1268 false negative detection.

\section{B. CONCLUSIONS}

Based on the result provided by the algorithms, the accuracy rate of other methods or combinations of values used for evaluation still needs further investigation since values for the parameters provided in the algorithms were manually calibrated, one basic example is the result provided by the Morphological Transformation, it can be observed in Figure 8 , that some fry were over eroded, which greatly affects the result of the counting since the basis of counting the 
candidates is based on contours detected, and in the image it can be observed that for some fry instead of one single fry, during erosion, some threshold values were not able to retain its original form instead it retains them separately. It can also be observed that false negative detection was heavy. And for those fry that tends to clumps at the near edge of the container, after erosion they were treated as one dark candidate, which would also yield one single count although it could be 4 or 5 or more fry.

\section{RECOMMENDATIONS}

Based on the results provided by the algorithms during the testing, image algorithm alone can find it difficult to yield a much higher acceptable result, in the sense that the algorithm has no prior knowledge in where they were applied or evaluated, with this, the proponent of this project recommends the following for future researchers whose interest is in Open Computer Vision and Artificial Intelligence:

1. Use of Machine Learning algorithm like Tensorflow is highly encouraged for it can help more the algorithm to have a prior knowledge of counts in a scenario were candidate's clumps, touched, or overlapped with each other.

2. Use of MATLAB is also encouraged to evaluate further the algorithms as MATLAB utilizes powerful matrix library.

3. Wide variety of Training set of different objects that way the algorithm will be more intelligent and will not rely only in a limited knowledge.

4. Methods for each algorithm must be further investigated in terms to what is the most accurate combination of values needed to produce an accurate and acceptable result.

5. Combination of different algorithms that focuses on segmentation.

\section{ACKNOWLEDGEMENT}

The researcher of this study fully acknowledges BFAR Reg 11 for providing the fry's involved in this study in the data collection stage, Commission on Higher Education under K-12 Scholarship Program for the scholarship and funding the research. The IT Team of SPAMAST-Digos campus for the wisdom, and undying support especially to Prof. Richie Boy Restauro, Prof. Darel Pulver, MIT, Klient Flores Gealon, and Jack Lord Canillo

\section{REFERENCES}

[1] Philippine Export Development Plan 2015-2017. http://www.philexport.ph/philippines-economy

[2] Regional Office for Asia and the Pacific. Status and potential of fisheries and aquaculture in Asia and the Pacific 2008. http://www.fao.org/docrep/011/i0433e/I0433E04.htm

[3] Westling, F. A., Sun, C., Wang, D., \& Alam, F. I. (2015). Fish counting and measurement: A modular framework and implementation. Computer Vision and Pattern Recognition in Environmental Informatics. https://doi.org/10.4018/978-1-4666-9435-4.ch003

[4] Labuguen, R. T., Volante, E. J. P., Causo, A., Bayot, R., Peren, G., MacAraig, R. M., .. Tangonan, G. L. (2012). Automated fish fry counting and schooling behavior analysis using computer vision. Proceedings - 2012 IEEE 8th International Colloquium on Signal Processing and Its Applications, CSPA 2012, 255-260. https://doi.org/10.1109/CSPA.2012.6194729

[5] P. P. Guan and H. Yan, "BLOOD CELL IMAGE SEGMENTATION BASED ON THE HOUGH," Proceedings of the 2011 International Conference on Machine Learning and Cybernetics, Guilin, , 10-13 July, 2011.

[6] Y. o. Z. Xitao Zheng, “A Fish Population Counting Method Using Fuzzy,” IEEE, pp. 225-228, 2010.

[7] Labuguen, R. T., Volante, E. J. P., Causo, A., Bayot, R., Peren, G., Macaraig, R. M., Libatique, N. J. C., \& Tangonan, G. L. (2012). Automated fish fry counting and schooling behavior analysis using computer vision. 2012 IEEE 8th International Colloquium on Signal Processing and Its Applications. https://doi.org/10.1109/cspa.2012.6194729

[8] Guan, P. P., \& Yan, H. (2011). Blood cell image segmentation based on the Hough transform and fuzzy curve tracing. 2011 International Conference on Machine Learning and Cybernetics. https://doi.org/10.1109/icmlc.2011.6016961

[9] Xitao Zheng, \& Yongwei Zhang. (2010). A fish population counting method using fuzzy artificial neural network. 2010 IEEE International Conference on Progress in Informatics and Computing. https://doi.org/10.1109/pic.2010.5687462 
Impact Factor $7.39 \div$ Vol. 11, Issue 1, January 2022

DOI 10.17148/IJARCCE.2022.11103

\section{BIBLIOGRAPHY}

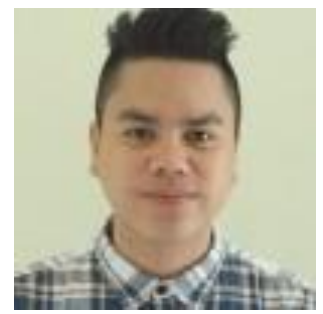

NEL R. PANALIGAN, MSIT is a former faculty of Southern Philippines Agri-Business And Marine and Aquatic School of Technology (SPAMAST) in Information Technology Department. Currently he is teaching in Davao del Sur State College under Computing Department. He conducted this research as his thesis project in graduate school of Ateneo de Davao University, where his research interest is into Artificial Intelligence, Computer Vision, and Image Processing. 